\title{
ADMIRÁVEL MOODLE NOVO. EXPERIÊNCIA DE REMODELAÇÃO DO AMBIENTE VIRTUAL DE APRENDIZAGEM E ATUALIZAÇÃO DO MOODLE.
}

RIO DE JANEIRO/RJ MAIO/2018

\author{
TELMA DE ALMEIDA SOUZA - INCA - tsouza@inca.gov.br \\ MONICA NOGUEIRA DA COSTA FIGUEIREDO - INCA - mfigueiredo@inca.gov.br \\ MÁRCIO DA SILVA CAMILO - INCA - marcio.camilo@inca.gov.br
}

Tipo: Relato de Experiência Inovadora (EI)

Categoria: Métodos e Tecnologias

Setor Educacional: EDUCAÇÃO SUPERIOR, EDUCAÇÃO CONTINUADA EM GERAL

\begin{abstract}
RESUMO
O presente trabalho é um relato de experiência sobre os desafios enfrentados no processo de remodelação do ambiente virtual de aprendizagem e na atualização do Moodle, da versão 1.9 para a versão 3.2, realizados pelo Núcleo de Educação a Distância do Instituto Nacional de Câncer. A remodelação e a atualização foram motivadas pela necessidade estratégica de oferecer uma plataforma moderna, acessível e segura para os cursos oferecidos. O processo teve como pontos importantes a criação de uma nova identidade visual para o ambiente; as configurações $e$ reprogramações de plugins do Moodle, para adaptá-los ao layout e comportamento desejados; e a criação de um curso piloto como prova de conceito. O curso criado foi submetido à avaliação de uma turma piloto e, após um processo de depuração de questões relacionadas a funcionalidades e ao layout, a plataforma foi considerada pronta para ser utilizada.
\end{abstract}

Palavras-chave: Moodle, upgrade, plugins, identidade visual, ambiente virtual de aprendizagem. 


\section{Introdução}

O Instituto Nacional de Câncer (INCA) qualifica profissionais para atuarem na prevenção e controle do câncer no país e, cada vez mais, utiliza as tecnologias educacionais interativas, para superação de limitações geográficas, temporais e financeiras. Assim, são desenvolvidas atividades que complementam os processos de ensino e possibilitam o contato com outros centros e com profissionais de diferentes localidades e/ou regiões mais remotas. O Núcleo de Educação a Distância (NEAD) do INCA gerencia essas atividades e utiliza o software Moodle como plataforma de ensino.

Por meio de estudos sobre a efetividade das ações educacionais no Ambiente Virtual de Aprendizagem (AVA), a equipe do NEAD concluiu que a realização de uma atualização (upgrade) no Moodle e a remodelação da identidade visual do AVA, seriam requisitos estratégicos para a melhoria da qualidade dos cursos ofertados e o consequente aumento na adesão por parte dos alunos. No presente trabalho, apresentamos o processo de atualização do Moodle e de remodelação do AVA, a experiência obtida ao longo do processo e as perspectivas futuras, enfatizando os desafios e as estratégias de enfrentamento realizadas.

\section{Referencial teórico}

Ambientes virtuais de aprendizagem (AVA) são softwares, normalmente do tipo web, ou seja, disponíveis pela internet, para usuários que podem acessá-lo de diferentes localidades (Martins, Tiziotto e Cazarini, 2016; Ribeiro, Mendonça e Mendonça, 2007; Bertini e Carneiro, 2015). Apresentam um conjunto de interfaces e ferramentas tecnológicas que possibilita a construção de redes de informação e conhecimento. Outras nomenclaturas podem ser encontradas como: ambientes virtuais de ensinoaprendizagem (AVEA), ambientes digitais de aprendizagem Learning Management System (LMS), enfatizando a faceta gerencial do ambiente ou Virtual Learning Environment (VLE), ênfase no processo de ensino-aprendizagem (Colling, Moesch e Schmitz, 2017; Pereira, 2007; Anjos, 2012).

Um AVA deve ser bem planejado e projetado para permitir que o processo de ensinoaprendizagem aconteça de acordo com estratégias educacionais bem definidas (Beluce e Oliveira, 2012). Costa e Franco (2005) observam que um web-site educacional, onde informações são disponibilizadas, não é necessariamente um AVA. Tampouco o AVA deve ser uma reprodução cibernética de uma sala de aula real (Haddad, 2013). Dessa forma, em um AVA a autonomia deve ser preconizada, ou seja, os estudantes devem poder escolher seus próprios caminhos para a construção de conhecimento. 
Os AVAs possuem formas de comunicação que propiciam uma diminuição do fator de distância. A comunicação entre tutores e estudantes e entre estudantes entre si pode se dar de forma síncrona ou assíncrona (Haddad, 2013). Tais ambientes também suportam a criação de trabalhos que podem ser desenvolvidos de forma colaborativa e cooperativa entre os estudantes com a supervisão dos tutores (Silva, 2009). AVAs podem ser utilizados em atividades presenciais, semipresenciais e em cursos a distância. Apresentam como vantagens: interação entre aluno e conteúdo, possibilidade de se dar atenção individualizada a um aluno, controle do próprio ritmo de aprendizagem pelo aluno, apresentação de conteúdo de modo criativo, atrativo e integrado e a possibilidade de ser configurado para permitir a avaliação do aluno (Ribeiro, Mendonça e Mendonça, 2007).

As ferramentas disponibilizadas em um AVA podem ser agrupadas em eixos (Silva, 2009): 1. Informação e documentação: download e upload de arquivos, páginas de conteúdo e interfaces; 2. Comunicação: síncrona (bate papo online) e assíncrona (fórum); 3. Gerenciamento pedagógico e administrativo: resultados de avaliações, registros de atividades, coordenação virtual de um curso; 4. Produção: Tarefas como estudos de caso e resolução de problemas disponíveis no ambiente. É possível integrar múltiplas mídias, linguagens e recursos apresentando informações de forma organizada, desenvolvendo interações e socializando produções a fim de atingir os objetivos propostos (SILVA, 2007). Além disso, um AVA pode estabelecer uma região de potencialidade para o aprendizado, representando o que é chamado por Vygotsky de Zona de Desenvolvimento Proximal (Mozzer, 2009). Assim, o docente deixa de ser aquele que simplesmente transmite o conhecimento para se tornar um mediador agindo como elo no processo de construção de conhecimento. Conteudista, tutor e aluno tornam-se atores cooperativos no processo de ensino e de aprendizagem e, dessa forma, desenvolvem-se e constroem novos conhecimentos (Bertini e Carneiro, 2015). Esse ambiente é baseado na teoria socioconstrutivista fundamentada na concepção de que os alunos aprendem melhor quando estão envolvidos em um processo social de construção de conhecimento (Taborda, 2007).

O Moodle é um AVA de código aberto, ou seja, pode ser copiado e modificado, com restrições, como manutenção da licença e dos copyrights originais. Seu nome é um acrônimo de Modular Object-Oriented Dynamic Learning Environment (Bertini e Carneiro, 2015). É um software modular, no qual diversos recursos e atividades existentes podem ser adicionadas a um curso de acordo com os objetivos que norteiam a ação educativa (Leite, 2006). Para funcionar precisa ser instalado em um servidor web, computadores próprios ou empresa de hospedagem (Pereira e França, 2013). Na sua infraestrutura estão envolvidos servidores web (apache, IIS entre outros), 
interpretador e linguagem de back-end (PHP), bancos de dados relacionais (MySQL, PostgreSQL entre outros) e linguagens de front-end (HTML, CSS e Javascript), que são interpretadas e renderizadas em um navegador web como o Google Chrome, o Mozilla Firefox ou o Internet Explorer. A comunidade Moodle, formada por pessoas e instituições que adotaram esse AVA, conta com cerca de 48.000 membros de aproximadamente 115 países. A comunidade brasileira é bastante ativa, atua no aperfeiçoamento da plataforma e contribui com discussões relevantes e trabalhos colaborativos para melhoria do ambiente.

\section{Justificativa da atualização}

Em 2016, o AVA do INCA era baseado no Moodle na sua versão 1.9. Apesar de considerada uma versão madura do Moodle e apresentar suas principais funcionalidades (Rice IV, 2008), as novas funcionalidades disponibilizadas ao longo dos anos não estão disponíveis ou satisfatórias na versão 1.9. Algumas dessas funcionalidades estão disponíveis no core (parte central, essencial ou básica de uma aplicação) das novas versões do Moodle, como por exemplo funcionalidades de acompanhamento e conclusão de etapas de um curso, emblemas (badges), novos modelos de exercícios do tipo quiz e aderência a modernas plataformas de design gráfico e visual como o uso da biblioteca bootstrap (Büchner, 2016).

Outras funcionalidades são acessíveis através da instalação de plugins (Henrick e Holland, 2015), como: barras de progresso, opções avançadas de emissão de certificados, temas mais modernos e customizáveis, entre outros. Além das funcionalidades encontradas no core e também em plugins, seria necessário dispor de plugins de temas, para realizar a remodelação visual do AVA, que não estavam disponíveis para a versão 1.9 do Moodle. Outro ponto importante considerado é a necessidade de possuir o software sempre atualizado para que as questões de segurança estejam sempre devidamente resolvidas (Miletic, 2011). Finalmente, era preciso ter uma versão atualizada para que se pudesse ter acesso a um suporte mais qualificado por parte da comunidade do Moodle. Na medida em que a versão 1.9 foi se defasando, ficou cada vez mais difícil se encontrar quem pudesse tirar dúvidas ou compartilhar experiências sobre essa versão do Moodle. Esses fatores fundamentaram a realização do upgrade para a versão 3.2 do Moodle.

\section{Desafios da atualização}

O primeiro e talvez o maior desafio encontrado para a realização da atualização é de ordem técnica e diz respeito ao fato de que não há como migrar o Moodle da versão 1.9 
diretamente para a versão 3.2. Isso porque houve grandes alterações entre essas duas versões e o recomendável nestes casos é que se faça primeiro um upgrade para a versão 2.2, depois para a versão 2.7 e finalmente para a versão desejada de acordo com os guias do Moodle. Além disso, como o NEAD possui cerca de 20 cursos oferecidos e outros em vários estágios do processo de desenvolvimento (desde concepção até testes com turmas piloto no AVA) não seria possível realizar essa migração imediata de todos os cursos para a nova versão do Moodle sem que essa migração interrompesse o curso ou causasse algum transtorno à sua execução.

Além disso, após análise sobre os temas disponíveis, constatou-se que nenhum deles se adequava exatamente a identidade visual que tinha sido concebida para a nova versão do AVA. Também alguns plugins apresentavam funcionalidades ou identidade visual que eram incompatíveis com o que se desejava para a nova versão do AVA. Com relação a funcionalidades a serem implementadas, mais um desafio foi encontrado, porque alguns plugins precisavam ser adaptados, seja por configuração ou seja por reprogramação, para a melhor visualização de conteúdos nas páginas principais e dentro dos livros e para a adequação de questões de avaliação.

\section{Soluções}

Com relação a arquitetura, em um trabalho em conjunto com a equipe de Tecnologia de Informação do INCA, foi definida uma solução de transição entre as duas versões do Moodle. A versão 1.9 seria mantida em um servidor de produção enquanto um servidor de desenvolvimento e teste foi disponibilizado para a criação da nova versão do AVA utilizando o Moodle na versão 3.2, sendo, posteriormente, criado um servidor de produção para o Moodle na versão 3.2. Durante um tempo teremos duas plataformas sendo trabalhadas paralelamente, em produção: uma com o Moodle 1.9 servindo como histórico de todos os cursos ministrados e outra com o Moodle 3.2 com os novos cursos sendo produzidos e oferecidos nela.

Com relação a adaptação do tema ao design visual, foi necessário realizar um processo de reprogramação do plugin do tema escolhido. O tema escolhido foi o Roshnilite (https://moodle.org/plugins/theme_roshnilite) e algumas alterações em folhas de estilos e templates foram realizadas para que se obtivesse o resultado desejado. Configurações e até reprogramações em outros plugins foram necessárias para que se pudesse adaptar as funcionalidades e a identidade visual àquela que foi concebida para a nova versão do AVA. Assim, foram realizadas configurações e reprogramações nos plugins livro, para apresentação de conteúdo e quiz, para o formato de questões de questionários de avaliação. O plugin do livro foi reprogramado para possibilitar a apresentação de 
conteúdo de forma mais limpa e suave sem a apresentação das barras de Navegação e Administração. A figura 1, a seguir, mostra o livro sem as abas laterais.

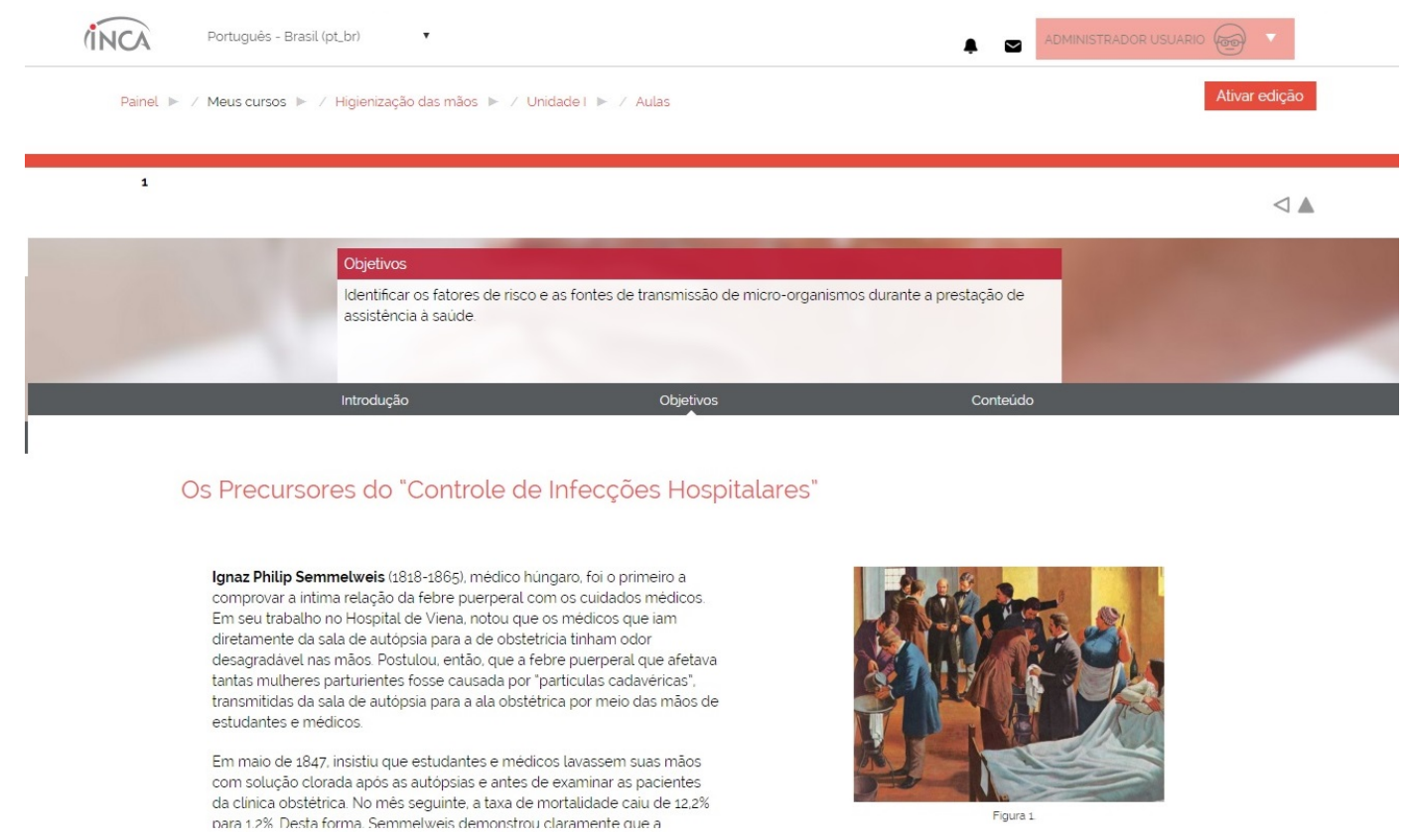

Figura 1. Livro do Moodle sem as abas laterais de Navegação e Administração

Após a instalação e configurações de tema do Moodle 3.2 no ambiente de desenvolvimento, migrou-se um curso para novo AVA para serem realizados mais testes com relação às funcionalidades e ao design visual. $O$ curso escolhido chama-se Higienização das Mãos e ele foi escolhido por ser um curso com conteúdo menor (carga horária de 4 horas). O processo de desenvolvimento no curso de Higienização das Mãos no novo AVA com o Moodle 3.2 foi realizado em paralelo com a carga de trabalho dos cursos em produção (no Moodle 1.9). Todo o conteúdo do curso foi redesenhado para essa nova versão de forma que se adequasse bem ao projeto visual da nova plataforma. Esse curso possui em sua avaliação, duas questões que possuem gráficos. Na figura 2, podemos ver a comparação entre as possibilidades de questões do quiz do Moodle na versão 1.9 e na 3.2 . 

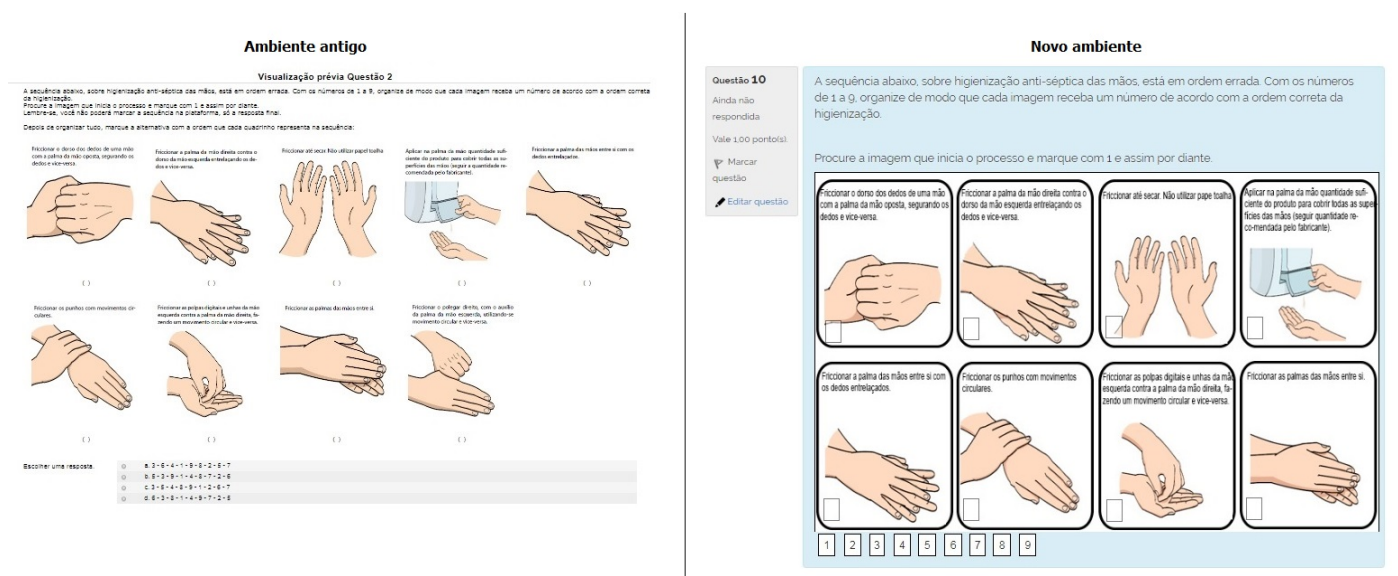

Figura 2. Questão sobre higienização das mãos na versão 1.9 e na versão 3.2 do Moodle

$\mathrm{Na}$ forma como são apresentadas na plataforma antiga, havia dificuldade por parte dos alunos, já que precisavam anotar os números para descrever a ordem correta dos passos de higienização e então escolher a opção que traduz corretamente essa ordem. Com as novas possibilidades de questões do quiz do Moodle na versão 3.2 foi possível reconstruir essas questões para que ficassem mais intuitivas, utilizando-se a modalidade de arrastar e soltar sobre imagem. Porém, como o funcionamento do quiz só permite imagens com as dimensões máximas de 600 pixels de largura por 400 pixels de altura, foi necessário reprogramar o plugin para aumentar esses limites e ter imagens maiores onde se pode ler o texto contido nelas.

\section{Estágio atual}

Com o novo curso criado foi estabelecida uma turma piloto que realizou diversas observações sobre o funcionamento da nova plataforma. Essas observações foram listadas e tornaram itens a serem solucionados pela equipe do NEAD. A cada item foi atribuído um status e uma descrição do problema a ser resolvido e o profissional por quem ele devia ser tratado. Todos os itens foram resolvidos e testados pelos membros da equipe. Na figura 3, podemos ver a tela inicial do curso de Higienização das Mãos. $O$ curso possui um Guia do Aluno e 5 unidades de conteúdo. Possui também uma avaliação e a possibilidade de emissão do certificado. Além disso, no curso é possível acompanhar o andamento com a ferramenta de acompanhamento de completude e com a barra de progresso. Também é possível o aluno obter emblemas na medida em que cumpre etapas do curso. 


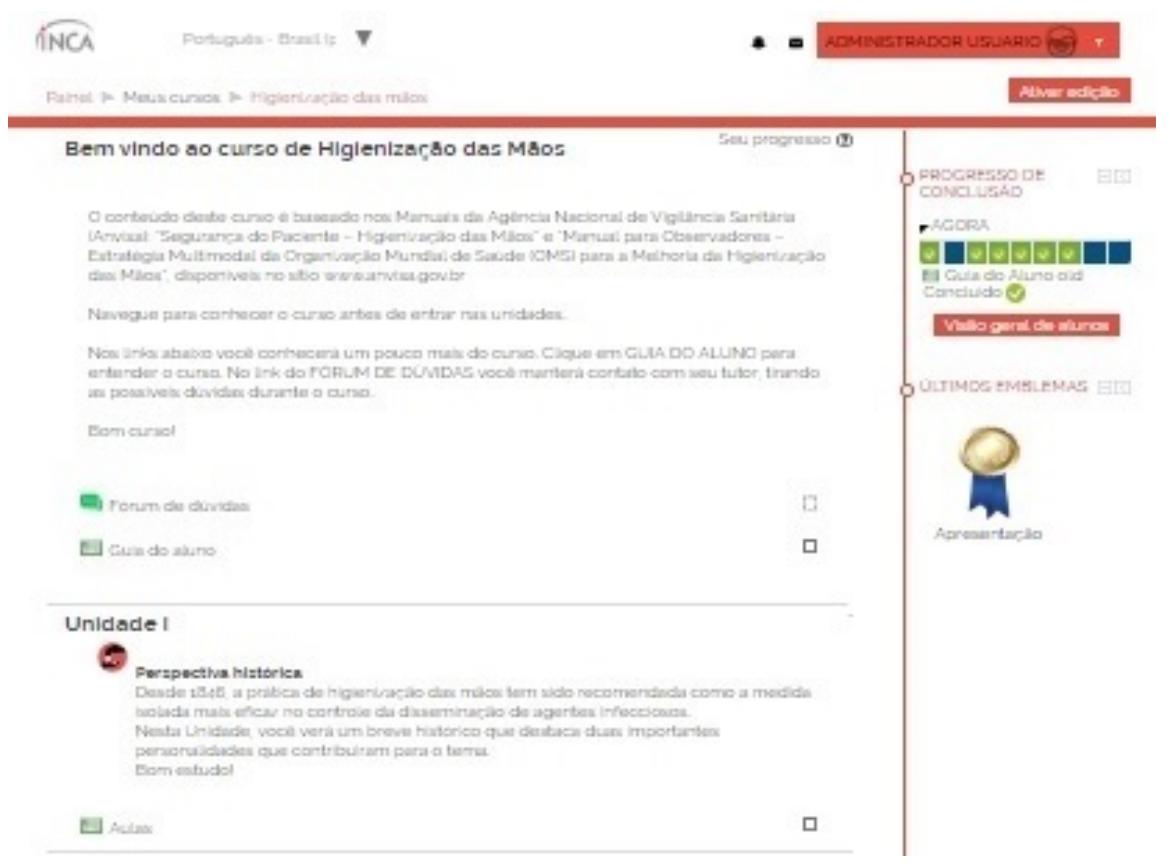

Figura 3. Tela inicial do curso de Higienização das mãos com Moodle 3.2

\section{Perspectivas futuras}

A partir da criação e disponibilização de um servidor de produção pela equipe de Tecnologia de Informação, os demais cursos serão desenvolvidos no novo ambiente, para poderem ser acessados pelos discentes dos cursos do INCA. Também será realizada a capacitação dos tutores, para que compreendam os acessos e ferramentas da nova plataforma e possam acompanhar os alunos, desenvolvendo as diversas funções da tutoria no ambiente atualizado.

\section{Conclusão}

O presente trabalho apresentou a experiência de remodelação do Ambiente Virtual de Aprendizagem e de atualização do Moodle realizados pelo Núcleo de Educação a Distância do INCA.

Foram apresentados os desafios envolvidos no processo e as soluções propostas. A dificuldade de atualização do Moodle entre versões muito díspares foi resolvida com a adoção de dois servidores rodando as versões diferentes do AVA em paralelo. O tema Roshnilite foi escolhido e adaptado para proporcionar a identidade visual do AVA desejada. Plugins foram utilizados e adaptados (configurados e reprogramados) para que as funcionalidades necessárias pudessem ser disponibilizadas.

A modularidade e versatilidade do Moodle têm se mostrado como poderosas aliadas, 
permitindo que o progresso no processo de remodelação e atualização ocorra de forma viável, sustentável e segura. Espera-se que o novo ambiente melhore a experiência de interação para alunos e professores e que a sistematização e divulgação da estratégia realizada possam colaborar na experimentação e desenvolvimento de processos similares por outras instituições.

\section{Referências}

Anjos, A. M. Tecnologias da informação e da comunicação, aprendizado eletrônico e ambientes virtuais de aprendizagem. In: Maciel, C. Ambientes Virtuais de Aprendizagem. Cuiabá: EdUFMT, p. 11-58, 2012.

Beluce, A. C.; Oliveira, K. L. Ambientes virtuais de aprendizagem: das estratégias de ensino às estratégias de aprendizado. In: IX ANPED SUL, p. 1-14, 2012.

Bertini, L.; Carneiro, R. F. A comunicação no ambiente virtual de aprendizagem de um curso a distância para formação de professores. Revista Educação em Questão, Natal, v.52, n. 38, p. 137-162, maio/agosto, 2015.

Büchner, A. Moodle 3 Administration. An administrator's guide to configuring, securing, customizing, and extending Moodle. 3를 edição. Birmingham: Packt Publishing, 2016.

Colling, J; Moesch, B.; Schmitz, L. L. O uso de plataforma moodle no processo de orientação de trabalhos de conclusão de curso e estágios. In: XXIII Congresso Internacional de Educação a Distância, Foz do Iguaçu, 2017.

Costa, L. A. C.; Franco, S. R. K. Ambientes virtuais de aprendizagem e suas possibilidades construtivistas. In: Congresso Global de Educação em Engenharia e Tecnologia, Santos, 2005.

Haddad, M. Ambientes Virtuais de Aprendizagem (AVAs) no ensino presencial e semipresencial de graduação da UFSJ. 2013. Dissertação (Mestrado em Educação) Universidade Federal de São João Del-Rei. São João Del-Rei.

Henrick, G; Holland, K. Moodle Administration Essentials. Learn how to set up, maintain, and support your Moodle site efficiently. Birmingham: Packt Publishing, 2015.

Leite, M. T. M. O ambiente virtual de aprendizagem Moodle na prática docente: conteúdos pedagógicos. Laboratório de Educação a Distância. UNIFESP, 2006. 
Martins, D. O.; Tiziotto, S. A.; Cazarini, E. W. Ambientes Virtuais de Aprendizagem (AVAs) como ferramentas de apoio em Ambientes Complexos de Aprendizagem (ACAs). Revista Brasileira de Aprendizagem Aberta e a Distância, v. 15. ABED, 2016

Miletic, D. Moodle Security. Learn how to install and configure Moodle in the most secure way possible. Birmingham: Packt Publishing, 2011.

Mozzer, L. D. Ambientes virtuais de aprendizagem: conceitos e estratégias de comunicação. In: Biblioteca virtual do NEAD/UFJF, 2009. Disponível em: http://www.cead.ufjf.br/wp-

ontent/uploads/2015/05/media_biblioteca_ambientes_virtuais_conceitos.pdf. Acesso em 14 mar. 2018.

Pereira, A. T. C.; S. V.; Dias M. R. A. C. AVA - Ambientes Virtuais de Aprendizagem em Diferentes Contextos. Rio de Janeiro: Editora Ciência Moderna Ltda, p. 4-22, 2007.

Pereira, L. S. S. A.; França, G. Os ambientes virtuais de aprendizagem (ava): um estudo do moodle no curso de pedagogia da UFT. In: Revista Científica Internacional, ed. 25, v. 1, art. 5, 2013.

Ribeiro, E. N.; Mendonça, G. A. A.; Mendonça, A. F. A importância dos ambientes virtuais de aprendizagem na busca de novos domínios da EAD. In XIII Congresso Internacional da Associação Brasileira de Educação a Distância, Curitiba, 2007.

Rice IV, W. H. Moodle 1.9 E-Learning Course Development. A complete guide to successful learning using Moodle 1.9. Birmingham: Packt Publishing, 2008.

Silva, L.; Silva, M. A avaliação online num ambiente virtual de aprendizagem. In: V Encontro de Educação e Tecnologias de Informação e Comunicação, 2007.

Silva, I. M. M. Ambiente virtual de aprendizagem na educação a distância. In: Congresso Nacional de Ambientes Hipermídia para Aprendizagem. Florianópolis, 2009.

Taborda, M. Ambientes virtuais de aprendizagem na educação superior: da teoria à prática. In XIII Congresso Internacional da Associação Brasileira de Educação a Distância, Curitiba, 2007. 